GEOPHYSICAL RESEARCH LETTERS, VOLUME 20, NUMBER 13, PAGES 1331-1334, JULY 9, 1993

\title{
SELF-CONSISTENT ELECTROSTATIC POTENTIAL DUE TO TRAPPED PLASMA IN THE MAGNETOSPHERE
}

\author{
Ronald H. Miller and George V. Khazanov \\ Space Physics Research Laboratory, Department of Atmospheric, Oceanic and Space Sciences \\ University of Michigan
}

\begin{abstract}
A steady state solution for the self-consistent electrostatic potential due to a plasma confined in a magnetic flux tube is considered. A steady state distribution function is constructed for the trapped particles from the constants of the motion, in the absence of waves and collisions. Using Liouville's theorem, the particle density along the geomagnetic field is determined and found to depend on the local magnetic field, self-consistent electric potential, and the equatorial plasma distribution function. $\mathrm{A}$ hot anisotropic magnetospheric plasma in steady state is modeled by a biMaxwellian at the equator. The self-consistent electric potential along the magnetic field is calculated assuming quasineutrality, and the potential drop is found to be approximately equal to the average kinetic energy of the equatorially trapped plasma. The potential is compared with that obtained by Alfvén and Fälthammar [1963].
\end{abstract}

\section{Introduction}

Alfven and Fulthammar [1963] were the first to study the generation of an electrostatic potential structure due to trapped particles in a magnetic mirror (magnetic flux tube). They demonstrated that an anisotropic collisionless plasma in a magnetic field can be in quasineutral equilibrium without a parallel electric field only if the magnetic field is homogeneous or if the pitch-angle anisotropy is the same for both electrons and ions. They considered a single particle approach and determined that the electrostatic potential energy difference $($ le $\mid \varphi)$ between the ionosphere $\left(B=B_{j}\right)$ and equator $\left(B=B_{0}\right)$ is on the order of the mirror ratio $\gamma \sim B_{i} / B_{0}$ multiplied by the mean perpendicular particle energy. For a typical mirror ratio $(\gamma \sim 40$ for an $L$ shell of 4 and an altitude of $2000 \mathrm{~km}$ ), the potential energy determined by Alfvén and Fälthammar [1963] can be well in excess of the total kinetic energy of the trapped plasma which creates this potential.

In steady-state, Persson [1963] evaluated the particle distribution function using Liouville's theorem and confirmed the results of Alfven and Fälthammar [1963]. However, Persson [1963] never computed the total potential necessary for quasineutrality when the electrons and ions have different anisotropies. Whipple [1977] considered a generalization of the work by Alfvén and Fälthammar [1963]. The integral formalism of Whipple [1977], using a delta function for the trapped plasma at the equator, reproduces the results of Alfvén and Fälthammar [1963]; however, the electric potential, due to a variety of trapped distributions (in the absence of other plasma sources), was not investigated and compared to the Alfvén and Fälthammar [1963] model. Chiu and Schulz [1978] considered a self-consistent treatment for the electrostatic potential; however, their analysis included ionosphetic plasma extracted upward by parallel electrostatic fields and backscattered electrons. Chiu and Schulz [1978] state that the Alfvén and Fälthammar [1963] model would produce unrealistically large potential drops (on the order of several hundred $\mathrm{keV}$ ), but they never discuss the validity of the Alfven and Fälthammar [1963] model. They do, however, mention that the large electric potentials would decrease in the presence of ionospheric plasma. Chiu and Schulz [1978] found potential drops on the order of the plasma kinetic energy; however, it is unclear from their analysis as to the magnitude of the electrostatic potential in the absence of ionospheric plasma and backscattered electrons.

In this paper, we construct a steady-state distribution function, in the absence of waves and collisions, from the kinematic constants of the motion, namely the total energy and the magnetic moment. Using Liouville's theorem, the local density for species $j$ is given in terms of an integral over the equatorial distribution function for that species, where the limits of integration are determined from the constants of the motion. The limits of integration and, consequently, the particle density are found to depend on the self-consistent electrostatic potential and the local magnetic field. For a given equatorial distribution function, the electric potential is determined through the condition of quasineutrality. The self-consistent treatment for the electric potential is compared to the electric potential determined by Alfvén and Fälthammar [1963]. We will show three main results in this paper. Firstly, we will show that the self-consistent theory produces potential drops approximately equal to the total kinetic energy of the equatorially trapped plasma. Secondly, we will show that the Alfvén and Fälthammar [1963] (hereafter referred to as the A-F model) model predicts electric potentials which are bounded and do not scale generally as the magnetic mirror ratio times the average kinetic energy of the trapped particles (le $\left.\mid \varphi \neq \gamma W_{\mathrm{ave}}\right)$. Not only is the electric potential from the A-F model bounded, but at the mirror points (which corresponds to the maximum potential possible), the potential is independent of the magnetic mirror ratio and is proportional to the average kinetic energy of the trapped plasma. Thirdly, we will demonstrate that for a bi-Maxwellian distribution for electrons and ions, the A-F model produces a potential which agrees within a factor of 2 (at the average mirror point) with that from the self-consistent theory; however, the spatial dependence from both theories are very different. This difference will be shown to come from the single-particle formalism of the A-F model, which loses information concerning the finite velocity spread and infinite number of reflection points characterized by a bi-Maxwellian.

\section{Theory}

\section{Kinetic Model}

Since the plasma is considered to be collisionless, the timeindependent distribution function depends only on the constants of the motion, namely, the particle's total energy

$$
E=\frac{m_{j}}{2}\left(v_{\|}^{2}+v_{\perp}^{2}\right)+e_{j} \varphi
$$

and magnetic moment

$$
\mu=\frac{m_{j} v_{\perp}^{2}}{2 B}
$$


where $e_{j}$ and $m_{j}$ are the species charge and mass, $v_{\|}$and $v_{\perp}$ are the parallel and perpendicular velocities, $\varphi$ is the electric potential, and $B$ is the magnetic field. The potential difference can be related to the parallel electric field from the following relation

$$
\varphi(s)=-\int_{0}^{3} E_{\|}\left(s^{\prime}\right) d s^{\prime}
$$

where $E_{\| l}$ is the parallel electric field and where the potential at the equator is assumed to be zero $(\varphi(s=0)=0, s=0$ is the equator).

From Liouville's theorem, the particle distribution is constant along any particle trajectory which is characterized by the constants of the motion; therefore, the distribution function at any point along the geomagnetic field can be calculated provided it is known at the equator (since we have an equatorially trapped plasma). For convenience, we will transform our distribution function and integration variables from $\left(v_{\|}, v_{\perp}\right)$ to constants of the motion $(\mu, E)$. The particle density for species $j$ can then be written as

$$
\begin{aligned}
n_{j} & =\frac{\pi B}{2}\left(\frac{2}{m_{j}}\right)^{3 / 2} \iint d E d \mu \frac{\left(f_{j+}(\mu, E)+f_{j-}(\mu, E)\right)}{(E-e, \varphi-\mu B)^{1 / 2}} \\
& =\frac{\pi B}{2}\left(\frac{2}{m_{j}}\right)^{3 / 2} \iint d E d \mu \frac{2 f_{j}(\mu, E)}{\left(E-e_{j} \varphi-\mu B\right)^{1 / 2}}
\end{aligned}
$$

which is similar to that found by Whipple [1977] and where $f_{j+}$ and $f_{j-}$ are the particle distributions for $v_{\|}>0$ and $v_{\|}<0$, respectively. Using the total energy $(E)$ and magnetic moment $(\mu)$, we have lost the distinction between particles moving in opposite directions along the magnetic field. For trapped particles, however, we can invoke symmetry between both directions and included a factor of two in the integrand of Eq.(4).

The particle density, at arbitrary positions along the magnetic field, is easily determined provided the distribution function is known at the equator. The region of determination for the integrals (limits of integration), in Eq. (4), is obtained by considering the condition of reflection which occurs for $v_{\|}=0$ and is given by

$$
v_{\| l}=\left(\frac{2}{m_{j}}\right)^{1 / 2}\left(E-e_{j} \varphi\left(s^{\prime}\right)-\mu_{j} B\left(s^{\prime}\right)\right)^{1 / 2}=0
$$

which is a function of the total equatorial energy, the local electric potential and magnetic field, and the sign of the charged particle. Equation (5) depends only on the final position $\mathbf{s}^{\prime}$ and is a necessary but not sufficient condition to guarantee accessibility of an equatorial particle to s'. For particles to gain access to s', they must not be reflected at intermediate positions $\left(0<s<s^{\prime}\right)$ along the magnetic field. This introduces a constraint on the functional dependence of the electric potential with respect to the magnetic field and was discussed in detail by Chiu and Schulz [1978]. They found two constraints on the electric potential

$$
\begin{gathered}
\frac{d \varphi}{d B}>0 \\
\frac{d^{2} \varphi}{d^{2}} \leq 0
\end{gathered}
$$

which must be valid at all positions between the equator and $\mathrm{s}^{\prime}$ (see Chiu and Schulz [1978] for more details). Equations (6) and (7) require the potential to increase monotonically with $B$ throughout the entire region $0<s<s^{\prime}$. Thus, any solution of Eq.(4), where Eqs.(6) and (7) are not valid, must be discarded, since it would be based on a false mapping of the distribution function in $\mathrm{Eq} .(4)$.

Provided Eqs.(6) and (7) are valid, the condition of reflection at $s^{\prime}$ yields $E=e_{j} \varphi\left(s^{\prime}\right)+\mu_{j} B\left(s^{\prime}\right)$ where particles having $E<e_{j} \varphi\left(s^{\prime}\right)+\mu_{j} B\left(s^{\prime}\right)$ are reflected before reaching $s^{\prime}$. Particles with $E \geq e_{j} \phi\left(s^{\prime}\right)+\mu_{j} B\left(s^{\prime}\right)$, reach $s^{\prime}$ and contribute to the particle density $n_{j}\left(s^{\prime}\right)$. Using the limits of integration, the particle density for species $j$ can be written as

$$
\mathrm{n}_{\mathrm{j}}=\frac{\pi \mathrm{B}}{2}\left(\frac{2}{\mathrm{~m}_{\mathrm{j}}}\right)^{3 / 2} \int_{\mu^{\prime}=0} \int_{\mathrm{E}^{\prime}=\mathrm{e}, \mathrm{g} \varphi(s)+\mu_{\mathrm{j}} \mathrm{B}(z)} \mathrm{dEd} \mu \frac{2 f_{\mathrm{j}}(\mu, E)}{\left(E-\mathrm{e}_{\mathrm{j}} \varphi-\mu \mathrm{B}\right)}
$$

and depends on the electric potential, magnetic field and equatorial distribution function. Since we have assumed a zero potential at the equator, the total energy at the equator is only the kinetic energy which is positive definite. Hence, for our application, caution must be used so that the lower bound on the energy integral is over positive energies (i.e., for $\mu=0$ and $\left.e_{j} \varphi(s)<0, E^{\prime}<0\right)$. For this reason, we introduce a Heaviside function into the equatorial distribution, forcing the integration over positive energies regardless of the electric potential. With a zero electric potential at the equator, the bi-Maxwellian distribution function can be written as

$$
\begin{aligned}
f_{j}(\mu, E) & =n_{0}^{2}\left(\frac{m_{j}}{2 \pi}\right)^{3 / 2}\left(\frac{1}{T_{j 110} T_{j \perp 0}^{2}}\right)^{1 / 2} \\
& \times \exp \left(-\frac{E}{T_{j 10}}-\frac{\mu B_{0}}{T_{j 110}}\left(\frac{T_{j 110}}{T_{j \perp 0}}-1\right)\right) H(E)
\end{aligned}
$$

where $n_{0}$ is the equatorial density, $T_{j \| 0}$ is the parallel thermal energy $\left(T_{j \|}=m, v_{j \|}^{2} / 2\right), T_{j \perp 0}$ is the perpendicular thermal energy $\left(T_{j L}=m_{j} v_{j L}^{2} / 2\right), H(E)$ is a Heaviside function, and $B_{0}$ is the equatorial magnetic field (note: the subscript 0 throughout this paper denotes equatorial values). Given the functional form of the equatorial distribution function, the particle density, at arbitrary positions along the magnetic field, can be written as

$$
n_{j}=n_{0} \frac{\exp \left[-\phi\left\{1-H\left(-\phi \frac{T_{j 100}}{T_{j \perp 0}}\right)\left[1-\frac{B_{0}}{B}\left\{1-\frac{T_{j 110}}{T_{j \perp 0}}\right\}\right]\right\}\right]}{\left(\frac{T_{j \perp 0}}{T_{j 110}}+\frac{B_{0}}{B}\left\{1-\frac{T_{j \perp 0}}{T_{j 10}}\right\}\right)}
$$

where $\phi=e_{j} \varphi(s) / T_{j l 10}$. The expression multiplying the Heaviside function in Eq. (10) only contributes to the exponential if $\phi<0$ due to the nature of the Heaviside function.

From the assumption of quasineutrality, $\mathrm{n}_{\mathrm{e}}(\varphi)=\mathrm{n}_{\mathrm{i}}(\varphi)$, and using Eq.(10) for the densities, the electrostatic potential becomes

$$
\frac{|e| \varphi(s)}{T_{\text {ello }}}=A_{0} \cdot \operatorname{Ln}\left[\frac{\frac{T_{e \perp 0}}{T_{\text {ell }}}+\frac{B_{0}}{B}\left\{1-\frac{T_{e \perp 0}}{T_{\text {ell0 }}}\right\}}{\frac{T_{i \perp 0}}{T_{i l l 0}}+\frac{B_{0}}{B}\left\{1-\frac{T_{i \perp 10}}{T_{i 110}}\right\}}\right]
$$


with

$$
\begin{gathered}
\left(A_{0}\right)^{-1}=1+\frac{T_{\text {ell0 }}}{T_{\text {illo }}}-H\left(\phi_{e}\right)\left[1-\frac{B_{0}}{B}\left\{1-\frac{T_{\text {ello }}}{T_{\text {eL10 }}}\right\}\right]- \\
\frac{T_{\text {ell0 }}}{T_{\text {ill0 }}} H\left(-\phi_{i}\right)\left[1-\frac{B_{0}}{B}\left\{1-\frac{T_{\text {ello }}}{T_{\text {e10 }}}\right\}\right]
\end{gathered}
$$

where $\phi_{j}=\left|e_{j}\right| \varphi(s) / T_{j l \mid 0}$. Equation (11) guarantees quasineutrality for various equatorial electron and ion anisotropies. In the limit of a homogenous magnetic field or if the electrons and ions have the same anisotropy, Equation (11) produces a zero electric potential. Subsequently, only for unequal electron and ion anisotropies is an electric potential generated and, subsequently, an electric field.

\section{Alfvén and Fälthammar Model}

The A-F model calculates the total electrostatic potential drop along the magnetic field using a single particle approach (see Alfvén and Fülthammar [1963] page 165) and can be written as

$$
\text { lel } \varphi=(\gamma-1)\left[\frac{\mathrm{W}_{\mathrm{ill0}} \mathrm{W}_{\mathrm{c} \perp 0}-\mathrm{W}_{\mathrm{i} \perp 0} \mathrm{~W}_{\text {ell1 }}}{\mathrm{W}_{\mathrm{ill0}}+\mathrm{W}_{\mathrm{ellO}}}\right]
$$

where $\gamma=B_{i} / B_{0}$ is the ratio of the magnetic field strength at the ionosphere to equator, $W_{j \| 0}\left(1 / 2 \mathrm{~m}_{\mathrm{j}} \mathrm{v} \|^{2}\right)$ and $W_{j \perp 0}$ $\left(1 / 2 m_{j} v_{\perp}{ }^{2}\right)$ are the parallel and perpendicular energies at the equator. From Eq.(8), and assuming quasineutrality, the selfconsistent integral model reproduces the results of A-F [Eq.(12)] for a delta function distribution in energy and magnetic moment [Whipple, 1977].

The total potential drop, given by Eq.(12), is on the order of the mean perpendicular energy of the plasma times the magnetic mirror ratio $\left(\gamma=B_{j} / B_{0}\right)$. For large mirror ratios, the total potential drop can be in excess of the total plasma kinetic energy which would seem to violate energy conservation; however, implied in the analysis and not explicitly found in Eq.(12) is that the analysis is invalid above (toward ionosphere) the mirror point of the particles. This condition restricts the magnitude of the potential and can be easily seen by the following considerations. From conservation of energy, the total energy at the mirror point can be related to the total energy at the equator from the following relationship,

$$
W_{j \perp m}+e_{j} \varphi_{m}=W_{j \perp 0}+W_{j l 0}+e_{j} \varphi_{0}
$$

where $m$ refers to the mirror point, $W_{\perp}$ and $W_{\|}$are the perpendicular and parallel energies and where we have assumed $W_{j l l m}=0\left(v_{i}=0\right)$. Considering electrons and ions, the electric potential can be removed from Eq.(13) to produce the following relationship

$$
\mathrm{W}_{\mathrm{ellO}}+\mathrm{W}_{\mathrm{ill0}}=\left(\frac{\mathrm{B}_{\mathrm{m}}}{\mathrm{B}_{0}}-1\right)\left(\mathrm{W}_{\mathrm{eL} 10}+\mathrm{W}_{\mathrm{iL0}}\right)
$$

Inserting Eq.(14) into Eq.(12), we obtain a relationship for the A-F electric potential at the mirror point

$$
|e| \varphi_{m}=\left[\frac{W_{i 100} W_{e \perp 0}-W_{i \perp 0} W_{e \| 10}}{W_{i \perp 0}+W_{e \perp 0}}\right]
$$

which is independent of the mirror ratio. Assuming the electron energy is in the perpendicular direction $\left(\mathrm{W}_{\mathrm{ell}}=0\right)$ and the ion energy is in the parallel direction $\left(\mathrm{W}_{\mathrm{i} \perp 0}=0\right)$, the electrostatic potential drop becomes proportional to the ion parallel energy, lel $\varphi \sim W_{\text {illo }}$. Hence, the A-F model conserves energy up to the mirror point, Eq.(15). Above the mirror point $\left(s>s_{\text {mirror }}\right.$ ), the A-F model [Eq.(12)] no longer conserves energy and produces unreasonably large electric potentials, in excess of the total plasma energy, as will be shown in following section.

\section{Results}

We found in Section 2 that the self-consistent and A-F models are identical for a delta function distribution; however, the models are generally not identical for various trapped particle distributions. In this section, results are investigated using a bi-Maxwellian for the equatorial plasma. The average parallel and perpendicular energies for the plasma are calculated at the equator, and the potential from the A-F model is determined [Eq.(12)] along the geomagnetic field. Using the average parallel and perpendicular temperatures associated with the bi-Maxwellian plasma, the electric potential at the mirror point [Eq.(15)] is determined. The potential from the self-consistent model [Eq.(11)] is then compared to that calculated from the A-F model.

Figure 1 shows two curves, for the electric potential in $\mathrm{keV}$, as a function of normalized distance along the geomagnetic field line. Since the electric potential is symmetric about the equator, only half of the magnetic field line is shown, while the equator is designated by $\mathrm{s}=0$. For an $\mathrm{L}=4$ geomagnetic field line, $4 \mathrm{R}_{\mathrm{E}}$ from the equator toward the ionosphere corresponds to an altitude of approximately $1900 \mathrm{~km}$ in the ionosphere. We have chosen, for the electrons and ions, biMaxwellian distributions with the following temperatures and anisotropies: $\mathrm{T}_{\mathrm{el|}}=25 \mathrm{keV}, \mathrm{T}_{\mathrm{i} \mid \mathrm{l}}=25 \mathrm{keV}, \mathrm{T}_{\mathrm{e} \perp} / \mathrm{T}_{\mathrm{ell}}=2, \mathrm{~T}_{\mathrm{L} L} / \mathrm{T}_{\mathrm{i} \mid \mathrm{l}}=1$ (note: for equal electron and ion anisotropies both theories yield the same vanishing electric field along the magnetic field). The A-F potential ( $\varphi_{\text {A.F}}$, solid curve) is exponential in nature and at the ionosphere has a maximum value of 552 $\mathrm{keV}$. The potential drop from the A-F model, at the ionosphere, is much larger than the total plasma energy at the equator, scaling as the magnetic mirror ratio times the average energy at the magnetic equator lel $\varphi \sim{ }^{\prime} W_{\text {ave. }}$.

The A-F model, however, characterizes each particle with an average parallel and perpendicular energy calculated from the equatorial distribution function; therefore, an average

\section{Electrostatic Potential}

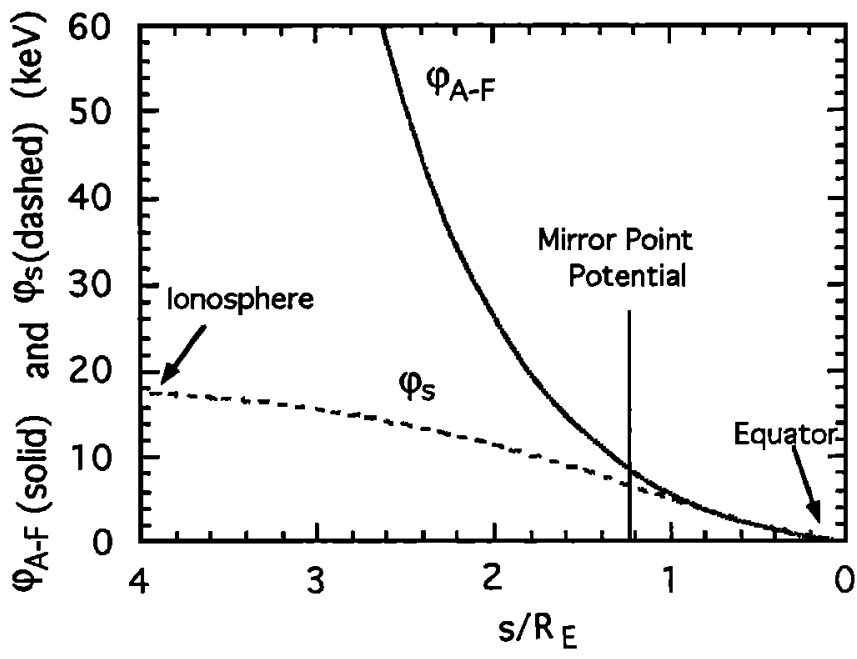

Fig. 1. The electric potential for the Alfvern and Fälthammar (solid) and self-consistent (dashed) models as a function of normalized distance along an $L=4$ flux tube for an equatorial distribution function with the following parameters $T_{\text {ell }}=25$ $\mathrm{keV}, \mathrm{T}_{\mathrm{ill}}=25 \mathrm{keV}, \mathrm{T}_{\mathrm{e} \perp} / \mathrm{T}_{\mathrm{ell}}=2, \mathrm{~T}_{\mathrm{i} L} / \mathrm{T}_{\mathrm{ill}}=1$. 
mirror point is associated with all the particles. The electric potential, at the average mirror point, is determined from Eq.(15) and is found to be $8.3 \mathrm{keV}$ for this case. This electric potential corresponds to a spatial location of $1.2 \mathrm{R}_{\mathrm{E}}$ from the equator and is on the order of the average energy of the equatorially trapped plasma [Eq.(15) and Figure 1]. Since no particles exist above the average mirror point $\left(s>1.2 R_{E}\right)$, the A-F model is not valid for distances greater than $1.2 R_{E}$. The assumption that the magnitude of the electric potential is approximately the mirror ratio time the average energy is valid only for distance below (toward equator) the mirror point $\left(s<s_{\text {mirror }}\right)$. The A-F model conserves energy, for distance smaller than $1.2 \mathrm{R}_{\mathrm{E}}$, and produces potentials approximately equal to those from the self-consistent model. The self-consistent potential ( $\varphi_{s}$, dashed curve) shows a very gradual increase in magnitude off the equator and has a maximum value of $18 \mathrm{keV}$ at the ionosphere. The information of a distribution of particles in velocity space is lost in the A-F formalism, while this information is an integral part of the self-consistent model. Because of this fundamental difference, the A-F potential increases more rapidly off the equator and explains the divergence between the two potentials.

\section{Summary}

We have constructed a time-independent distribution function for each species, in the absence of waves and collisions, from the kinematic constants of the motion, enabling a self-consistent treatment for the generation of an electrostatic potential due to trapped particles in a magnetic flux tube. A relationship for the density of each species (electrons and ions) was derived that can be expressed in terms of an integral over the species equatorial distribution function. Integration limits were determined, from the constants of the motion, which depend on the local potential and magnetic field. Through the condition of quasineutrality, an electrostatic potential was found for trapped electrons and ions with different anisotropies.

Three main results were obtained in this paper. The first result is that the self-consistent electrostatic potential [Eq.(12)], due to an equatorially trapped plasma in the absence of thermal ionospheric plasma, is on the order of the mean kinetic energy of the trapped plasma. A simple physical scenario as to why this result is reasonable comes from considering a plasma confined in a gravitational field. A polarization electric field develops which offsets the charge separation due to the different masses of the particles. For this case, the electric force is approximately half the gravitational force $\left(T_{0}=T_{i}\right)$, i.e., the electric potential is on the order of the gravitational potential. The source of energy, for this instance, is from the gravitational field, while for the case presented in this paper, the free energy can only come from the total energy of the plasma. We chose a bi-Maxwellian distribution for the trapped particles; however, we have investigated the electric potential due to a number of different trapped distributions and found similar results as those using a bi-Maxwellian. In all cases, the electrostatic potentials are on the order of the kinetic energy of the plasma.

The second result is that the A-F potential scales as the magnetic mirror ratio times the average plasma kinetic energy only in a limited region of configuration space, centered about the equator. The A-F model is valid provided the particle density is nonzero, which is true for distance below the mirror point (toward equator). Using conservation of energy, the electrostatic potential at the average mirror point of the particles [Eq.(15)] was determined, removing the dependence on the magnetic mirror ratio. The potential at the mirror point is the largest potential possible given the magnetic field geometry and the total plasma energy. The mirror point potential, therefore, is an upper bound on the magnitude of the A-F potential. Thus, the general statement that the A-F potential scales as the magnetic mirror ratio time the average plasma kinetic energy [Alfvén and Fälthammar, 1963; Persson, 1963, 1966;, Block and Falthammar, 1976; Chiu and Schulz, 1978] must be modified to include the spatial region of applicability.

The third result concerns the application of the A-F model for particle distributions which are not delta functions in energy and magnetic moment. We were able to use the A-F model for a bi-Maxwellian by determining the average parallel and perpendicular energies for the electrons and ions. The electric potential for both cases diverge slightly near the equator $\left(s<1.2 R_{E}\right)$. Above the mirror point (toward ionosphere, $s>1.2 R_{E}$ ), the A-F potential exponentially increased to values well in excess of the total plasma kinetic energy; however, the self-consistent potential showed a gradual increase toward the ionosphere. The discrepancy between these theories is due to the average single particle nature of the A-F model, while the self-consistent theory allows for a distribution of velocities and an infinite number of reflection points.

Acknowledgments. The authors wish to thank T. Gombosi, J. Kozyra, A. Nagy, T. Neubert, C. Rasmussen, and D. Winske for useful discussions. This work was supported at the University of Michigan by the National Aeronautics and Space Administration (NASA) grant NAGW-1619 and by the National Science Foundation under contract ATM-9111440.

\section{References}

Alfvén, H., and C.-G. Fülthammar, Cosmical Electrodynamics, Fundamental Principles, Oxford University Press, London, 1963.

Block, L. P., and C.-G. Fulthammar, Mechanisms that may support magnetic-field-aligned electric fields in the magnetosphere, Ann. Geophys., 32, 161-174, 1976.

Burke, W. J., and M. Heinemann, Origins and consequences of parallel electric fields, Solar-Terrestrial Physics, edited by R. L. Carovillano and J. M. Forbes, D. Reidel Publishing Company, 1983.

Chiu, Y. T., and M. Schulz, Self-consistent particle and parallel electrostatic field distributions in the magnetospheric-ionospheric auroral region, J. Geophys. Res., 83, 629-642, 1978.

Persson, H., Electric field along a magnetic line of force in a low-density plasma, Phys. Fluids, 6, 1756, 1963.

Persson, H., Electric field parallel to the magnetic field in a low density plasma, Phys. Fluids, 9, 1090, 1966.

Stern, D., The electric field and global electrodynamics of the magnetosphere, Rev. Geophys. and Space Sci., 17, 626$640,1979$.

Whipple, E. C., Jr., The signature of parallel electric fields in a collisionless plasma, J. Geophys. Res., 82, 1525-1531, 1977.

R. H. Miller and George V. Khazanov, Space Physics Research Laboratory, Department of Atmospheric, Oceanic and Space Sciences, University of Michigan, Ann Arbor, MI 48109-2143.

(Received November 17, 1992; revised March 31, 1993; accepted April 23, 1993.) 